\title{
Gedächtnis und Erinnerung
}

Alle Lebewesen sind um des Überlebens willen auf eine biologische Merkfähigkeit angewiesen, die es ihnen ermöglicht, Wahrnehmungsreize zu ,speichern“, sie in irgendeiner Form zu verarbeiten und je nach Bedarf spontan zu wiederholen oder ,abzurufen“. Von dieser genetisch verankerten Merkfähigkeit unterscheidet sich das, was unsere Sprache mit den Worten „Erinnerung und Gedächtnis“ und was andere europäische Sprachen mit Vokabeln benennen, die von dem lateinischen Wort memoria abgeleitet sind, das seinerseits von griechisch mnéme abstammt. In allen Fällen verweisen die Wörter auf innere, sei es spontane, sei es willentliche Denk- und Vorstellungstätigkeiten, die sich allerdings im Unterschied zu anderen kognitiven bzw. imaginativen Akten auf zeitlich frühere Wahrnehmungen oder Gedanken beziehen und das Moment der Wiederholbarkeit (iteratio) einschließen. ,Schon unsere Sprache gibt dem Gedächtnis“, notiert Hegel in der „Enzyklopädie der philosophischen Wissenschaften“, ,die hohe Stellung der unmittelbaren Verwandtschaft mit dem Gedanken“ (\$ 464).

In der europäischen Ideengeschichte ist das Erinnerungsvermögen sehr früh als anthropologisches Apriori, nämlich als eine Bedingung der praktischen wie theoretischen Erfahrung verstanden worden und zählt insofern auf allen Stufen der Tradition zu den allgemein anerkannten Voraussetzungen des leiblich-geistigen Retentionsvermögens. Im Dialog „Menon“ (80a-82a) läßt Platon durch Sokrates den Beweis führen, alles Erkennen sei Wiedererinnern (anámnesis). Der Platonschüler Aristoteles diskutiert in der kleinen Schrift „De memoria et reminiscentia" ausführlich Funktion und Leistung des Erinnerungsvermögens im Verhältnis zur Wahrnehmung und zum Denken. Seine vermögenspsychologisch grundierten Überlegungen geben für Jahrhunderte den Ton in der einschlägigen Theoriebildung an. Ihre Quintessenz gerinnt zu folgenden Aussagen:

- „Gedächtnis“ (memoria) und „Erinnern“ (reminiscentia) verhalten sich zueinander wie das langsamere (animalische) zum schnelleren (intelligenten) Behalten (449b), anders gesagt: wie das passive Affiziertwerden der Sinne zu den aktiven Bewegungen des Denkvermögens (453a).

- Wahrnehmung bezieht sich auf Gegenwärtiges, Voraussicht auf Zukünftiges, Gedächtnis auf das Vergangene; allein das Gedächtnis stimmt mit dem Zeitsinn überein (449b, 451a).

- Voraussetzung für das Erinnern ist der Eindruck, den eine frühere „Wahrnehmung" oder „Meinung" als „Affektion“ oder „Habitus" im Subjekt hinterlassen hat; eine Spur, die sich in die Seele einzeichnet wie das Bild ins Wachs „, beim Siegeln mit dem Ringe“ (450a).

- Das früher Wahrgenommene sowohl wie das früher Gedachte werden eidetisch erinnert, will sagen: Objekt der Erinnerung sind Vorstellungsbilder (phantásmata), nicht der Reiz, die Sache oder das vorige Gedachte selbst (450a). „Hier- 
aus sieht man, welchem Teil oder Vermögen der Seele das Gedächtnis angehört, demselben nämlich wie die Phantasie“ (450a).

- Es gibt ein spontanes und ein suchendes Erinnern. Das letztere läßt sich durch technische Verfahren schulen, die in der Topik, d. h. in der rhetorisch-dialektischen Heuristik (inventio), entwickelt worden sind: z. B. die methodische Suche nach den „Orten/Plätzen“ (tópoi/loci), deren sequentielle Folgen „feste (mnemotechnische) Ordnungen" bilden. Beispiele sind mathematische und alphabetische Reihen, aber auch Schlußfolgerungen im Sinne logisch strukturierter Satzreihen.

Das aristotelische Konzept warf Probleme auf, die in der Folgezeit immer wieder Gegenstand erinnerungsträchtiger Reflexionen geworden sind. Die in Punkt 5 angesprochene mögliche Disziplinierung des Erinnerungsvermögens hat zumindest zwei Seiten. Zum einen zeigt sie Verwandtschaft mit der Form rationaler Urteilsbildung, zum andern erinnert sie an die in der sophistischen Rhetorik ausführlicher behandelten technischen Grundlagen jener ars memorativa (=Mnemonik), die, vermittelt über römische, spätantike, mittelalterliche und humanistische Schriftkundige, in das neuzeitliche Wissenschafts- und Bildungssystem eingewandert sind (Yates 1990; Bolzoni 1991; Coleman 1992). Das Christentum verstand sich nach dem Abendmahlswort „Tut dies zu meinem Gedächtnis!“ (Paulus, 1. Kor. 11, 24) als Erinnerungsgemeinschaft, und die alte Metaphorik des Einschreibens hat sich, gestärkt durch die normative Mnemonik der religiösen Buchkultur, als geschichtsmächtiges Interpretament durchgesetzt. Die maßgebenden technischen Regeln für das Training sowohl der memoria als auch der korrelativ mit ihr verbundenen inventio, die beide in den Dienst schriftbewahrender und auslegender Tätigkeiten traten, stammten aus Ciceros „De inventione“ und aus der Rhetorik des sogenannten Auctor ad Herenmium. „La memoria“, schrieb 1613 der Spanier Juan de Aranda, ,es un escribano que vive dentro del hombre" (Rossi 1991, S. 35). Dieses Sinnbild kennzeichnet eine für die Neuzeit charakteristische Verschiebung von der passiven zur aktiven Funktion des Gedächtnisses. Es löst den Topos vom Siegelringabdruck im Wachsblock ab, der seit Platon (Theaetet, 191c-d) und Aristoteles in Umlauf war, um eine angeblich unvermittelte Einwirkung der sinnlichen Perzeption auf den seelischen Rezeptor zu umschreiben.

Die starke Gewichtung des reproduktiven Gedächtnisses in der rhetorisch fundierten Mnemonik hat jenen Theoretikern keine Ruhe gelassen, die mit der Suche nach dem Schöpferischen dem nachspürten, was - im cartesianischen Sinne - dem Einschreiben bis dahin ungedachter, eben neuer Zeichen auf der absichtlich gelöschten Gedächtnistafel (tabula rasa) entsprach. Das Imitationsprinzip in der künstlerischen und literarischen Ausbildung hielt sich bekanntlich weitgehend an die mnemotechnischen Regeln der Iteration. Aristoteles hatte zwar zwischen dem niederen (langsamen) und dem höheren (schnelleren) Gedächtnisbzw. Erinnerungsvermögen unterschieden und die Einbildungskraft an der Erzeugung reproduzierbarer Vorstellungsbilder beteiligt; die den normativen Anspruch des mimetischen Gedächtnisses um eines Neuen willen überwindende schöpferische Spontaneität lag aber außerhalb seines Gesichtskreises. Damit war ein christlicher, von der Schöpfungstheologie geprägter Aristoteliker wie der ehemalige Rhetoriklehrer Augustinus nicht zufrieden. Er stieß z. B. bei seiner Selbstbefragung im 10. Buch der „Confessiones“ auf das irritierende Prinzip, daß selbst das Vergessen im Gedächtnis behalten werde (memoria retinetur oblivio). Dieses 
Erinnern des Nicht-Erinnerns wird ihm zum Ausgangspunkt für eine Selbstvergewisserung, die mit Hilfe der reproduktiven Erinnerung Selbst-Vergessenheit ausschließt, aber zugleich an die Grenzen der Ichtransparenz und damit auf das stößt, woran jede endliche Wahrnehmung und Erkenntnis scheitert. Diese Grenze $\mathrm{zu}$ überschreiten, ist nach Augustinus gerade nicht mehr Sache der Erinnerung, sondern allein des Willens (voluntas), der die Seele davon abbringt, allein in sich selbst zu „lesen“. Die Annäherung an das absolute Prinzip der Schöpfung, an Gott, folgt demnach einem Weg, der das Gedächtnis durchquert, um es hinter sich zu lassen (De Trinitate XI).

Die Neubewertung der schöpferischen Potenz in allen (mechanischen, sprachlichen, ästhetischen) Künsten nach dem Siegeszug der Neuen Wissenschaften unter der Flagge Bacons, aber auch Vicos - schien nur im Zerschneiden des aristotelischen Organon möglich: Petrus Ramus trennte die Phantasie, Montaigne den Intellekt, Diderot die Vernunft vom Gedächtnisvermögen (Rossi 1991, S. 49 ff.; Diderot, Éléments de physiologie, Kap. XXXII). Was das Erinnerungsvermögen, der ,innere Schreiber" Arandas, in „Zeichen“, „Bildern“, „Inschriften“ festhält, das ist - in der sensualistischen Perspektive John Lockes - die notwendige Voraussetzung für alles Denken, Urteilen und Erkennen gerade auch im Sinne neuer Einsichten und Entdeckungen. Ohne Gedächtnis (memory) wäre das Bewußtsein leer, hätte die Reflexion, die Locke mit dem Erinnern (remembrance) gleichsetzt, keine Gegenstände. Auch wenn die Vorstellungsbilder (ideas) dem Gedächtnis in „verblassenden Farben" eingeschrieben sind, der Geist hat die Macht, sie aktiv erinnernd wieder ins Bewußtsein zu rufen. Genau auf dieser retentional-reproduktiven Fähigkeit beruht aber, was Locke an Beispielen aus der Mathematik und Newtons Physik belegt, die Möglichkeit, eine ,,vergangene Erkenntnis" wiederzubeleben, um sie zur Grundlage neuer Erkenntnisse werden zu lassen (An Essay Concerning Human Understanding II, 10.5; IV, 1.9).

Der Fortschritt positiven Wissens wird bald durch systematisches Vergessen erkauft, da jede wissenschaftliche Revolution mit der dogmatischen Setzung eines Neuanfangs das vorhergehende Wissen entwertet. In Literatur und Künsten, die mit dem Fortschritt nicht verrechenbar sind, äußerte sich die im Namen der Neuheit vollzogene Abwendung von der Autorität des traditionellen Gedächtnis- und Imitationsprinzips in der Figur des Genies, von dem Lessing, stellvertretend für viele Zeitgenossen, behauptete, es verdanke seinen Reichtum nicht dem ,erworbenen Vorrat seines Gedächtnisses, sondern ... seinem eigenen Gefühl“ (Hamburgische Dramaturgie, 34. Stück). Gedächtnis steht hier für die Autorität des Tradierten. Wie diesem die Macht zu nehmen sei, wurde in der „Psychologia empirica" Christian Wolffs mit Hinweis auf die produktive Freiheit der Erfindungskraft (facultas fingendi) beantwortet, die das Gegebene (die überkommenen Muster) dekomponiert, um durch spontanes Umschreiben bzw. kombinatorisches Umstrukturieren etwas Neues zu schaffen ( $\$ 149)$.

Die durch die aufgeklärte Traditionskritik unterstützte Abkoppelung der (modernen) Gegenwart von der Vergangenheit hat die Frage nach der Emanzipation des Bewußtseins von der normativen Macht des Gedächtnisses verschärft. Wolffs „Psychologia empirica“ ( $\$ \$ 144,204)$ hielt an der Macht der Ars mnemonica fest, während er gleichzeitig die facultas legendi als eine vom Gedächtnis relativ unabhängige Form kreativen Umgangs mit den von der Einbildungskraft erzeugten Bildern (phantasmata) beschrieb. Kant verwarf in der „Anthropologie“ (1.Teil, §34) die ars mnemonica unter Hinweis auf die bewußte Willkür des Gedächtnis- 
vermögens bei der Reproduktion früherer Vorstellungsbilder. Hegel verspottete das "Gerede vom Aufbewahren der besonderen Vorstellungen in besonderen $F i$ bern und Plätzen“ (Enzyklopädie § 453). Er interpretierte die „Erinnerung“ als ein In-Beziehung-Setzen zwischen den im „nächtlichen Schacht“ der Innerlichkeit aufbewahrten Bildern und jener Anschauung, durch die das denkende Ich verallgemeinernd von dem Besitz ergreift, was ihm äußerlich ist. Er faßt das Gedächtnis, im Unterschied zur Erinnerung, als „Übergang in die Tätigkeit des Gedankens, der keine Bedeutung mehr hat", der bildlos und daher ohne Inhalt ist (§ 464). In der Bestimmung der Leitbegriffe „Erinnerung und Gedächtnis“ als formelle, von jedem bestimmten Inhalt freie Bedingungen der Geschichtsreflexion wehrt sich Hegels Philosophie gegen jeden von der Tradition ausgehenden inhaltlichen Autoritätsanspruch, ohne die zur Geschichte gewordene Tradition jenem Paradoxon aktiven Vergessens auszuliefern, das Nietzsches Angriff auf die historische Bildung in der „Zweiten Unzeitgemäßen Betrachtung“, ,Vom Nutzen und Nachteil der Historie für das Leben", propagiert hat.

Es ist bemerkenswert, daß Hegels Gedächtnistheorie durch die Befunde der wissenschaftlichen Neurophysiologie gestützt wird. Während die empirische Psychologie noch am Modell des Informationsspeichers festhält (Baddeley 1986), konzentriert sich die Hirnforschung auf die formellen Struktureigenschaften der Retention und Reproduktion. Da sich das Gedächtnis im menschlichen Gehirn nicht lokalisieren läßt, sucht sie die Erinnerungsleistung aus dem Zusammenspiel aller bekannten Hirnfunktionen zu erklären (Rosenfield 1988). Die Annahme eines solchen Zusammenspiels widerspricht der Isolierung eines einzigen, genau bestimmbaren Gedächtnisfeldes. Was wir „Gedächtnis“ oder „Erinnerung “ nennen, ist daher weder von der körperlich vermittelten Raum- noch von der sprachlich vermittelten Zeitwahrnehmung noch von der begrifflichen Kategorienbildung (Verallgemeinerung) zu trennen. Wie das Ich seine Identität im Fluß der Erfahrungen und in der Interaktion mit anderen ständig reorganisiert, ebenso verändert sich die „dynamische Struktur“ des Gedächtnisses bei jedem Wahrnehmungsreiz stets aufs neue (Rosenfield 1992, S. 14 ff.). Bewußtsein und Erinnerung bilden in dieser Perspektive eine flexible Einheit. Der Akt des Erinnerns gilt ihr - worauf die Semantik von „Gedächtnis“, „Gedenken“ und „Innerlichmachen“ hinweist - als Form einer zugleich rezeptiven und kreativen Retentions- und Kombinationsleistung kognitiver sowie imaginativer Operationen. Die entsprechenden Kombinationszüge beziehen sich zwar auf kontextualisierte Wahrnehmungen, doch "speichern" wir gerade nicht irgendwelche „Wahrnehmungsbilder", sondern erkennen, im Vollzug des in wechselnden Kontexten proteisch sich wandelnden Erinnerns, die Kategorien wieder, die während zeitlich früherer Erfahrungen ausgebildet worden sind: ,Our recognition of paintings or of people is the recognition of a category, not of a specific item. People are never exactly what they were moments before, and objects are never seen in exactly the same way" (Rosenfield 1988, S. 159).

Bleibt die Frage, ob diese Beobachtungen, die der traditionellen, von der Beziehung zwischen Gefäß (Speicher) und Inhalt (Information) ausgehenden Theorie eine offene Schema-Struktur des persönlichen Gedächtnisses vorziehen, auch für das Geltung besitzen, was Begriffe wie „kollektives“, „historisches" oder „kulturelles Gedächtnis“ umschreiben. Zunächst ist festzuhalten, daß die Entstehung der Geschichte als Gedächtniswissenschaft im Sinne von methodisch kontrollierten Archivierungs-, Konservierungs- und Aneignungstechniken mit der 
Evolution jener modernen, komplexen Gesellschaften zusammenfällt, die - anders als patrimonial strukturierte Gemeinschaften - ein reflektiertes Verhältnis zum Vergangenen besitzen. Es mag sein, daß sich das kulturelle, zunächst auf Mythogramme, Riten und orale Traditionen, in den Hochkulturen dann auf Schrift und schließlich auf andere technische bzw. elektronische Informationsspeicher gestützte Gedächtnis kollektiver Lebenswelten als eine stets komplexer werdende „Exteriorisierung“ des Kortex interpretieren läßt (Leroi-Gourhan 1988, S. 321 f.). Mit dem Reflexivwerden der Erinnerung im Hegelschen Sinne ist diese in Analogie zur Werkzeugerfindung konstruierte Hypothese nicht zu vereinbaren. Indessen gibt die Philosophie - in der Vermittlung von Vernunft und Geschichte - zu verstehen, daß mit dem Gang der Geschichte auch das historische Gedächtnis in die Zuständigkeit aktiven Handelns, hier des wissenschaftlich konstruierenden Denkens, fällt.

Das vergangene Geschehen kann nur historische Erinnerung werden, schreibt Droysen Mitte des 19. Jahrhunderts, wenn es , aus seiner Äußerlichkeit in den wissenden Geist und in dessen Kombinationen verlegt ist" (Droysen 1977, S. 8). Die historische Erinnerung ist also nicht gegeben. Was vom Vergangenen da ist, bedarf, da es in Bruchstücken vor Augen liegt, des methodischen Verstehens (der Interpretation), um Erinnerung, nämlich sinnhafte ,Totalität" zu werden. Wie Hegel geht es auch Droysen um die Freiheit vom Überkommenen, und ähnlich wie der Philosoph begreift er die historische Erinnerung als eine Gestalt der Reflexion: „Erst mit der Reflexion, in der wir es (das überkommene, unfreie Wissen) als vermitteltes erkennen, trennen wir es von uns selbst; die erkannte Tatsache der Vermittlung ist die Erinnerung; und diese Erinnerung trennen wir von uns selbst ... Erst damit beginnen wir, frei in uns selbst zu sein und mit dem, was unmittelbar unser Inhalt war, schalten zu können“ (Droysen 1977, S. 107). Das historische Gedächtnis ist demnach kein naturwüchsiges Mnemotop, in dem alles sinnvoll geordnet und abrufbar beieinander liegt. Es entsteht erst dann als ein „Gedankenbild“ und wird für die Gegenwart zur begriffenen, also geistig , lebendigen Erinnerung“, wenn es jener methodischen Recherche unterworfen worden ist, deren wissenschaftliche Grundlagen Droysens „Historik“ Schritt für Schritt entfaltet. Weder ist das historische „Faktum“ noch ist die ,Totalität” der Geschichte gegeben. Beides ist abhängig von einer konstruktiven Arbeit, die in zweifacher Bahn verläuft: in der Bahn der theoriegeleiteten Forschung und in der Niederschrift der historischen Darstellung.

Tradition erscheint unter den Bedingungen wissenschaftlicher Geschichtskonstruktion nicht mehr als Moment einer ungebrochenen, auf Dauer gestellten Kontinuität, sondern als eine Leitidee, unter deren Führung die Bruchstellen, über die hinweg Sinn ,getragen“ werden soll (tradere in der Ursprungsbedeutung von trans-dare), ins grelle Licht rücken. Denn die Bruchstellen sind, wie die inneren Widersprüche in einem Text, die Orte, an denen die Fragen aufgestellt sind, die der Interpret zu beantworten sucht. Droysens Begriff der „Totalität“, der auf den Sinn der Geschichte zielt, ist daher so abzuwandeln, daß auch die verpaßten Gelegenheiten als erinnernswerte Möglichkeiten unter ihn fallen. Die fragmentarische Synthese des historischen Gedächtnisses entsteht, mit den Worten Paul Ricoeurs, aus der dialektischen Bewegung zwischen „Sedimentierung“ (continuité) und „Innovation“ (discontinuité) (Ricoeur 1985, S. 395 ff.). Die Erinnerung spielt in diesem Prozeß das Amt des Vermittlers. Tradition, so auf Abstand gebracht, steht dann vielleicht als ein neuartiges theatrum memoriae, nämlich als ein Reper- 
toire von Geschichten zur Verfügung, über dessen Auswahl sich eine soziale Gruppe verständigen kann, um dem narrativen Kern ihrer politischen und kulturellen Identität das zuzurechnen, was sie für gut hält.

In komplexen Gesellschaften hat das historische Gedächtnis seine normative Geltung eingebüßt. Es deckt sich nicht mehr mit dem Gedächtnis des Kollektivs. Der Soziologe Maurice Halbwachs hat diesen Bruch in den 20er Jahren registriert und die identitätsbildende Kraft des kollektiven Gedächtnisses als „Tradition" von der Geschichte, die ,an dem Punkt beginnt, an dem die Tradition aufhört", unterschieden (Halbwachs 1985, S. 66). Ihm war durchaus bewußt, daß die zunehmende soziale Differenzierung auf das kollektive Gedächtnis einen permanenten Anpassungsdruck ausübt, und er hat sich nicht gescheut, die vom Kollektiv diesem unterstellte Beständigkeit als Illusion zu bezeichnen (Halbwachs 1985, S. 76, S. 163). Wenn aber das kollektive Gedächtnis eine imaginäre Struktur besitzt, die sich, dem Druck beschleunigter gesellschaftlicher und kultureller Differenzierungen nicht Stand haltend, in immer kürzeren Abständen dekomponiert und unter anderen Vorzeichen wieder aufbaut, dann sind umso mehr die Wissenschaften der historischen Gedächtniskonstruktion (unter Einschluß der Allgemein-, der Kultur- und Alltagsgeschichte) gefordert, das rettend zu bewahren, was im Rausch des Wandels verloren zu gehen droht.

\section{Literatur}

Aristoteles: Kleine naturwissenschaftliche Schriften (Parva naturalia), übers. v. E. Rolfes, Leipzig 1926. Assmann, A./Harth, D. (Hg.): Mnemosyne. Formen und Funktionen der kulturellen Erinnerung, Frankfurt/M. 1991. Assmann, J.: Das kulturelle Gedächtnis. Schrift, Erinnerung und politische Identität in frühen Hochkulturen, München 1992. Assmann, J./Hölscher, T. (Hg.): Kultur und Gedächtnis, Frankfurt/M. 1988. Baddeley, A.: So denkt der Mensch. Unser Gedächtnis und wie es funktioniert, München 1986. Berns, J. J./Neuber, W. (Hg.): Ars memorativa. Zur kulturgeschichtlichen Bedeutung der Gedächtniskunst 1400-1750, Tübingen 1993 (Frühe Neuzeit 15). Bolzoni, L.: The Play of Images. The Art of Memory from its Origins to the Seventeenth Century. In: Corti, P. (Hg.): The Enchanted Loom: Chapters in the History of Neuroscience, New York 1991, S. 16-26. Butler, T. (Hg.): Memory. History, Culture and the Mind, Oxford 1989. CAnciK, H./Mohr, H.: Erinnerung/Gedächtnis. In: Handbuch religionswissenschaftlicher Grundbegriffe II, Stuttgart 1990, S. 299-323. Carruthers, M. J.: The Book of Memory. A Study of Memory in Medieval Culture, Cambridge 1990. Cavalli, A.: Die Rolle des Gedächtnisses in der Moderne. In: Assmann, A./Harth, D. (Hg.): Kultur als Lebenswelt und Monument, Frankfurt/M. 1991, S. 200-210. Coleman, J.: Ancient and Medieval Memories. Studies in the Reconstruction of the Past, Cambridge 1992. Cook, P. (Hg.): Philosophical Imagination and Cultural Memory. Appropriating Historical Traditions, Durham/London 1993. Droysen, J. G.: Historik. Textausgabe v. P. Leyh, Stuttgart/Bad Cannstatt 1977. Ferraris, M.: Il fantasma della libertà. In: Iride VII, 11 (1994), S. 204-220. GAEchter, P.: Die Gedächtniskultur in Irland, Innsbruck 1970. HABERMAS, J.: Können komplexe Gesellschaften eine vernünftige Identität ausbilden? In: Habermas, J.: Zur Rekonstruktion des Historischen Materialismus, Frankfurt/M. 1976, S. 92-126. Halbwachs, M.: Les cadres sociaux de la mémoire, Paris 1925. Halbwachs, M.: Das kollektive Gedächtnis, 
Frankfurt/M. 1985. Harth, D. (Hg.): Die Erfindung des Gedächtnisses, Frankfurt/M. 1991. Haverkamp, A./Lachmann, R. (Hg.): Memoria. Vergessen und Erinnern (Poetik und Hermeneutik 15), München 1993. Hutton, P. H.: History as an Art of Memory, Hannover/London 1993. Le Goff, J.: Histoire et mémoire, Paris 1988. Leroi-Gourhan, A.: Hand und Wort. Die Evolution von Technik, Sprache und Kunst, Frankfurt/M. 1988. Metz, J. B.: Erinnerung. In: Krings, H/Baumgartner, H. M./Wild, C. (Hg.): Handbuch philosophischer Grundbegriffe, Bd.2, München 1973, S.386-396. Namer, G.: Mémoire et société, Paris 1987. Ricoeur, P.: Temps et récit III: Le temps raconté, Paris 1985. Rosenfield, I.: The Invention of Memory. A New View of the Brain, New York 1988. Rosenfield, I.: Das Fremde, das Vertraute und das Vergessene. Anatomie des Bewußtseins, Frankfurt/M. 1992. Rossi, P.: Il passato, la memoria, l'oblio, Bologna 1991. Schuster, M.: Zur Konstruktion von Geschichte in Kulturen ohne Schrift. In: Ungern-Sternberg, J. v./Reinau, H. (Hg.): Vergangenheit in mündlicher Überlieferung, Stuttgart 1988 (Colloquium Rauricum 1), S. 57-71. Y ATES, F. A.: Gedächtnis und Erinnern. Mnemonik von Aristoteles bis Shakespeare, Weinheim 1990. 